\title{
ASPECTOS FLORISTICOS E SÍNDROMES DE DISPERSÃO DAS ESPÉCIES ARBÓREAS DO MORRO DE SANTO ANTÃO, SANTA MARIA-RS ${ }^{1}$
}

Luis Fernando Alberti, José Augusto Nunes Hirt; Décio Brasil Flores Machado Junior, Mauricio Steckel e Cleandro Stevão Tombini Acadêmicos do Curso de Engenharia Florestal -CCR

UFSM - Santa Maria, RS

Solon Jonas Longhi

Departamento de Ciências Florestais - CCR

UFSM - Santa Maria, RS

\section{RESUMO}

O Morro de Santo Antão, localizado em Caturrita, no município de Santa Maria, além de ser um ponto turístico da região, abriga uma vegetação rica em espécies botânicas, pertencente à Floresta Estacional Decidual da Fralda da Serra Geral do Rio Grande do Sul. Obter informações sobre a composição florística e a síndrome de dispersão das espécies arbóreas foi o objetivo deste trabalho. A caracterização florística da floresta foi feita com detalhada identificação botânica de suas espécies. A síndrome de dispersão de sementes foi determinada através de observações no campo e revisão bibliográfica, seguindo os conceitos e categorias propostos por Van

\footnotetext{
${ }^{1}$ Pesquisa executada com Bolsa do CNPq/PIBIC
} 
Der Pijl, Howe \& Westley e Fisher \& Chapman e utilizados por NASCIMENTO \& LONGHI (1996). Foram encontradas 51 espécies arbóreas, distribuídas em 47 gêneros e 28 famílias botânicas, indicando considerável diversidade florística. As famílias Leguminosae, Myrtaceae, Sapindaceae e Moraceae foram as mais freqüentes da floresta. Entre as espécies, destacaram-se: Sorocea bonplandii (cincho), Cupania vernalis (camboatá-vermelho), Actinostemon concolor (laranjeira-do-mato), Schinus molle (aroeira-piriquita) e Nectandra megapotamica (canela-preta). $69 \%$ das espécies apresentaram dispersão zoocórica, confirmando a citação de vários autores que a consideram como a síndrome mais importante na disseminação das espécies arbóreas, bem como na manutenção do equilíbrio entre a flora e a fauna.

Palavras-chave: Composição florística, Floresta Estacional Decidual, Síndrome de dispersão de sementes.

\section{ABSTRACT}

The Santo Antão Hill located at Caturrita Village in the county of Santa Maria is an expressive tourist place in the region and also a rich forest in terms of botanic species from the lower montane Seasonal Deciduous Forests of the Serra Geral, Rio Grande do Sul. The objectives of the study were to get information about the floristic composition and seed dispersion syndromes of tree species. The floristic characterization of the Santo Antão hill forest was made with detailed taxonomic identification of the species found there. The seed dispersion was studied by field observations and bibliographical reviews using the categories and concepts proposed by Van Der Pijl, Howe \& Westley and Fisher \& Chapman and used by NASCIMENTO \& LONGHI (1996). There were 51 tree species classified in 47 genera and 28 families, indicating a relevant floristic diversity. Leguminosae, Myrtaceae, Sapindaceae and Moraceae were the most frequent families. Among the species, Sorocea bomplandii (cincho), Cupania vernalis (camboatá146 Rev. Ciência e Natura, Santa Maria, 22: $145-160,2000$. 
vermelho), Actinostemon concolor (laranjeira-do-mato), Schinus molle (aroeira-mansa), Nectandra megapotamica (canela-preta), were the most conspicuous. $69 \%$ of the studied species presented zoochoric dispersion, which is in agreement with severals authors that put this syndrome as one of the most important in the dissemination of tree species, as well as in the balance between the flora and fauna in the ecosystem.

Key words: Floristic composition, Seasonal Deciduous Forest, Seed Dispersal syndromes.

\section{INTRODUÇÃO}

A vegetação do Morro de Santo Antão, localizada na Fralda da Serra Geral do Rio Grande do Sul, pertence à Floresta Estacional Decidual (KLEIN, 1984; VELOSO et al., 1991), também conhecida como Floresta Subtropical Latifoliada (HUECK, 1972; RAMBO, 1956). A floresta Estacional Decidual ocorre na forma de disjunções florestais, apresentando o estrato dominante macro ou mesofanerofítico predominantemente caducifólio, com mais de $50 \%$ dos indivíduos despidos de folhagem no período desfavorável (VELOSO et al., 1991).

O Morro de Santo Antão está situado na localidade de Caturrita, no município de Santa Maria, Estado do Rio Grande do Sul. É considerado ponto turístico da região, por abrigar procissões religiosas (Festa de Santo Antão). Além disso, abriga uma floresta rica em espécies botânicas, importante para a manutenção da fauna local e constitui importante patrimônio genético natural.

O fato de existirem cultivos anuais nas encostas do Morro, bem como a ausência de algumas espécies de madeira nobre indica histórico de intervenção antrópica no local. Atualmente, o Morro de Santo Antão faz parte de uma área pertencente à Igreja Católica, sendo que grande parte de sua floresta ainda mantém-se relativamente preservada. 
Segundo MONTOYA MAQUIN \& MATOS (1967) a vegetação natural é uma comunidade muito complexa, que está relacionada com os diversos fatores do meio, como climáticos, edáficos e biológicos. Pode-se descrevê-la por diversos parâmetros, entre os quais destacam-se aqueles baseados no estudo dos diversos elementos da vegetação.

Para RIZZINI (1963), a caracterização de um tipo florestal pode ser fundamentada em caracteres florísticos, fisionômicos e ecológicos. O critério florístico identifica as espécies da floresta, o fisionômico descreve a sua aparência ou considera as características da paisagem que podem definir e diferenciar os diversos tipos de vegetação e o ecológico relaciona a vegetação com fatores do meio. Segundo KLEIN (1984) a Floresta da Fralda da Serra Geral tem altura de 20 a 30 metros e apresenta grande densidade de vegetação, lianas e epífitas. É muito heterogênea e rica em espécies de importância florestal (HUECK, 1972).

Alguns trabalhos florísticos e fitossociológicos da vegetação da Fralda da Serra Geral podem ser encontrados em SILVA \& LONGHI (1989), MACHADO \& LONGHI (1990) e LONGHI (1993), e servem de parâmetros comparativos para a floresta a ser estudada.

O estudo das síndromes de dispersão de sementes é prérequisito obrigatório para estudos na biologia evolutiva como por exemplo: competição entre espécies por dispersores, fuga de diásporas, dentre outros estudos. Para maiores esclarecimentos veja PIRES-O'BRIEN \& O'BRIEN (1995) e STEBBINS (1972).

TABARELLI et al. (1992) estudando a Floresta Estacional Decidual Baixo-montana (mata ciliar) no município de Santa Maria, encontrou mais de $70 \%$ das espécies arbóreas com síndrome de dispersão zoocórica, o mesmo acontecendo com NASCIMENTO \& LONGHI (1996), estudando um fragmento florestal no município de Candelária, Rio Grande do Sul. As espécies com síndrome anemocórica e autocórica estavam, principalmente, entre os grupos das pioneiras e secundárias iniciais. 
MORELLATO (1991), estudando uma floresta semidecídua no Sudeste do Brasil discute a interação entre a fenologia e a produção de frutos e sementes. Segundo a autora, os frutos de dispersão anemocórica e autocórica dispersam suas sementes na estação seca e os frutos de dispersão zoocórica são dispersos geralmente na estação chuvosa.

O número de espécies zoocóricas tende a crescer com a quantidade de chuva precipitada na estação chuvosa, ainda que espécies com frutos secos deiscentes de dispersão zoocórica, estejam competindo por dispersores, frutificando em diferentes épocas do ano. $57 \%$ das árvores e arvoretas apresentaram zoocoria naquele estudo.

O presente trabalho tem como objetivo obter informações sobre a composição florística e a síndrome de dispersão de sementes das espécies arbóreas visando fornecer subsídios a futuros estudos ecológicos e silviculturais na região.

\section{MATERIAS E MÉTODOS}

O presente trabalho foi realizado no Morro de Santo Antão, localizado no município de Santa Maria, a aproximadamente 480 metros altitude, $53^{\circ} 50^{\prime}$ de longitude $W$ e $29^{\circ} 37^{\prime}$ de latitude $S$.

O clima na região de estudos é do tipo Cfa (clima temperado úmido), de acordo com a classificação Climática de Köppen. O Estado do Rio Grande do Sul apresenta regime de chuvas variando de 1186 a $2468 \mathrm{~mm}$ anuais e a Depressão Central do Estado apresenta os menores valores tanto de precipitação como insolação anual quando comparada as outras regiões (LEMOS et alii, 1973). Segundo o mesmo autor Santa Maria possui solos do tipo Podzólico vermelho-amarelo, Latossolo vermelho-escuro distrófico e Brunizem hidromórfico, sendo que o morro em estudo possui afloramentos rochosos, solos litólitos distróficos de matriz basáltica e inclinação acentuada.

Para o estudo da composição florística foi realizado um 
trabalho de identificação botânica detalhada de suas espécies arbóreas componentes. As coletas de material botânico foram feitas mensalmente visando obter as fases de floração e frutificação. O material coletado no morro foi prensado, secado e identificado no laboratório de Dendrologia do Departamento de Ciências Florestais da Universidade Federal de Santa Maria. Este material encontra-se incorporado ao acervo do Herbário do Departamento de Ciências Florestais (HDCF).

Para determinar a sindrome de dispersão das espécies arbóreas, analisou-se detalhadamente a constituição dos frutos e sementes das mesmas, além de informações contidas em literaturas especializadas no assunto. Para sua determinação adotou-se a metodologia proposta por Van Der Pijl, Howe \& Westley, Fisher \& Chapman e NASCIMENTO \& LONGHI (1996), que classificaram as sementes em três grupos básicos de dispersão: anemocóricas, dispersas pelo vento; zoocóricas, dispersas por animais; e autocóricas, que não possuem morfologia própria para a dispersão em relação as categorias anteriores. Estas últimas diferenciam-se em espécies barocóricas e espécies de dispersão explosiva.

\section{RESULTADOS E DISCUSSÃO}

$\mathrm{Na}$ Tabela 1, podem ser observadas as espécies encontradas no local de estudo, com seus respectivos nomes científicos, nomes vulgares e famílias botânicas.

Foram encontradas 51 espécies distribuídas em 47 gêneros e 28 famílias botânicas (Tabela 2). Esse elevado número de espécies e gêneros é um indicativo da diversidade florística da floresta, confirmando a opinião de diversos pesquisadores, que citam a Floresta Estacional Decidual da Fralda da Serra Geral, como detentora de considerável diversidade, entre eles HUECK (1972), KLEIN (1984), SILVA \& LONGHI (1989), MACHADO \& LONGHI (1990) e LONGHI (1993). DIAS et al. (1996) encontraram em morros 
testemunha da Floresta da Fralda da Serra Geral em Santa Maria no Rio Grande do Sul, semelhante ao local estudado, uma alta diversidade florística, cujo valor do índice de Shanon foi de 3,60.

É necessário salientar, por outro lado, que estudos em áreas subtropicais (Mata Atlântica), na Floresta Estacional da Bacia do Alto Uruguai e na própria Floresta Estacional Decidual apresentaram diversidade superior.

Desta forma, qualquer consideração mais incisiva sobre as comparações entre diversidade são muito particulares e passíveis de serem influenciadas por diversos fatores.

Pode-se observar, pela Tabela 2, que as familias Myrtaceae, Fabaceae, Sapindaceae e Moraceae foram as mais destacadas na comunidade analisada. Destacam-se, também, as famílias Meliaceae, Euphorbiaceae, Flacourtiaceae, Boraginaceae, Mimosaceae, Rutaceae, Anacardiaceae, Lauraceae, Rosaceae e Bignoniaceae. As demais 14 famílias ( $50 \%$ do total) estão representadas por apenas 1 gênero e 1 espécie.

Trichilia, foi o gênero que apresentou maior número de espécies, sendo muito característico desse tipo de floresta e típico dos estratos inferiores. Allophylus e Eugenia também merecem destaque. Os demais 44 gêneros estão representados por apenas uma única espécie, comprovando a heterogeneidade de espécies no local estudado, com pouco domínio de alguns.

Os resultados da síndrome de dispersão das espécies arbóreas na floresta podem ser vistos na Tabela 3 , onde consta, também, a família botânica das mesmas. Nota-se, pela referida tabela, que a forma mais freqüente de dispersão de sementes foi a zoocórica, com aproximadamente $69 \%$ das espécies, o que confirma os resultados apresentados por vários autores que consideram-na como a mais importante síndrome de dispersão das espécies arbóreas na região, entre eles TABARELLI et al. (1992) e NASCIMENTO \& LONGHI (1996). 
TABELA 1: Nomes cientificos, nomes vulgares e famílias botânicas das espécies arbóreas encontradas na floresta do Morro de Santo Antão:

\begin{tabular}{|c|c|c|}
\hline NOME CIÊNTÍFICO & NOME VULGAR & FAMILIA \\
\hline Acnistus breviflorus Sendtn. & Esporão & Solanaceae \\
\hline Actinostemon concolor (Spreng.) Müll. Arg. & Laranjeira-do-mato & Euphorbiaceae \\
\hline Allophylus edulis (St. Hill.) Radlk. & Chal-chal & Sapindaceae \\
\hline Allophylus guaraniticus (St. Hill.) Radlk. & Vacum & Sapindaceae \\
\hline Bauhinia forficata Benth. & Pata-de-vaca & Caesalpiniaceae \\
\hline Carica quercifolia (St. Hill. ) Hieron. & Mamoeiro-do-mato & Caricaceae \\
\hline Campomanesia xantocarpa Berg & Guabiroba-do-mato & Myrtaceae \\
\hline Casearia sylvestris Sw. & Carvalinho & Flacourtiaceae \\
\hline Celtis iguanaea (Jacq.) Sarg. & Esporão-de-galo & Ulmaceae \\
\hline Chomelia obtusa Cham. Et Schlecht. & Viuvinha & Rubiaceae \\
\hline Cordia trichotoma (Vell.) Arrab. ex Steud. & Louro & Boraginaceae \\
\hline Chrysophyllum gonocarpum (Mart. \& Eich.) Engl. & Aguaí-da-serra & Sapotaceae \\
\hline Cupania vernalis Camb. & Camboatá-vermelho & Sapindaceae \\
\hline Dalbergia frutescens (Vell.) Britt. & Rabo-de-bugio & Fabaceae \\
\hline Diospyros inconstans Jacq. & Maria-preta & Ebenaceae \\
\hline Enterolobium contortisiliquum (Vell.) Morong & Timbaúva & Mimosaceae \\
\hline Erythrina falcata Benth. & Corticeira-da-serra & Fabaceae \\
\hline Eugenia involucrata DC. & Cerejeira-do-mato & Myrtaceae \\
\hline Eugenia uniflora L. & Pitangueira & Myrtaceae \\
\hline Ficus Iuschnathiana Miq. & Figueira-do-mato & Moraceae \\
\hline Helietta apiculata Benth. & Canela-de-veado & Rutaceae \\
\hline Hybanthus biggibosus (St. Hil.) Hassler & Fel-da-terra & Violaceae \\
\hline Lithraea molleoides (Vell.) Engler & Aroeira-brava & Anacardiaceae \\
\hline Luehea divaricata Mart. & Açoita-cavalo & Tiliaceae \\
\hline
\end{tabular}


TABELA 1 : Conclusão

\begin{tabular}{lll} 
Machaerium stipitatum Vogel & Canela-do-brejo & Fabaceae \\
Maclura tinctoria (L.) Don ex Steudel & Tajuva & Moraceae \\
Matayba elaeagnoides Radlk. & Camboatá-branco & Sapindaceae \\
Maytenus aquifolium Mart. & Cancorosa & Celastraceae \\
Myrcianthes pungens (Berg) Legr. & Guabijú & Myrtaceae \\
Myrocarpus frondosus Allem. & Cabriúva & Fabaceae \\
Nectandra megapotamica (Spreng.) Mez & Canela-preta & Lauraceae \\
Ocotea puberula Nees & Canela-guaicá & Lauraceae \\
Parapiptadenia rigida (Benth.)Brenan & Angico-vermelho & Mimosaceae \\
Patagonula americana L. & Guajuvira & Boraginaceae \\
Phytolacca dioica L. & Umbú & Phytolaccaceae \\
Plinia rivularis (Camb. ) Rotman & Guapuriti & Myrtaceae \\
Prunus sellowii Koehne & Pessegueiro-do-mato & Rosaceae \\
Quillaja brasiliensis Mart. & Pau-sabão & Rosaceae \\
Rollinia rugulosa Schlecht. & Araticum & Annonaceae \\
Schinus molle L. & Aroeira-piriquita & Anacardiaceae \\
Sebastiania commersoniana (Baill.) Smith \& Downs & Branquilho & Euphorbiaceae \\
Sorocea bonplandii (Baill.) Burger, Lanjow \& Boer & Cincho & Moraceae \\
Syagrus romanzoffiana (Cham.) Glassman & Jerivá & Arecaceae \\
Tabebuia heptaphylla Mart. & Ipê-roxo & Bignoniaceae \\
Tecoma stans (L.) Kunth & Caroba-louca & Bignoniaceae \\
Trichilia catigua A. Juss. & Catiguá-verdadeiro & Meliaceae \\
Trichilia claussenii C. DC. & Catiguá-vermelho & Meliaceae \\
Trichlia elegans A. Juss. & Catiguá-de-ervilha & Meliaceae \\
Vitex megapotamica (Spreng.) Mold. & Tarumâ & Verbenaceae \\
Xylosma pseudosalzmannii Sleumer & Espinho-judeu & Flacourtiaceae \\
Zanthoxylum rhoifolium L. & Mamica-de-cadela & Rutaceae \\
\hline
\end{tabular}


TABELA 2: Número de gêneros e espécies das diferentes famílias botânicas encontradas na floresta do Morro de Santo Antão:

\begin{tabular}{|c|c|c|}
\hline FAMILIA & $\mathrm{N}^{\circ} \mathrm{DE}$ GÊNEROS & $\mathrm{N}^{\circ}$ DE ESPÉCIES \\
\hline Anacardiaceae & 2 & 2 \\
\hline Annonaceae & 1 & 1 \\
\hline Arecaceae & 1 & 1 \\
\hline Bignoniaceae & 2 & 2 \\
\hline Boraginaceae & 2 & 2 \\
\hline Caesalpiniaceae & 1 & 1 \\
\hline Caricaceae & 1 & 1 \\
\hline Celastraceae & 1 & 1 \\
\hline Ebenaceae & 1 & 1 \\
\hline Euphorbiaceae & 2 & 2 \\
\hline Fabaceae & 4 & 4 \\
\hline Flacourtiaceae & 2 & 2 \\
\hline Lauraceae & 2 & 2 \\
\hline Meliaceae & 1 & 3 \\
\hline Mimosaceae & 2 & 2 \\
\hline Moraceae & 3 & 3 \\
\hline Myrtaceae & 4 & 5 \\
\hline Phytolaccaceae & 1 & 1 \\
\hline Rosaceae & 2 & 2 \\
\hline Rubiaceae & 1 & 1 \\
\hline Rutaceae & 2 & 2 \\
\hline Sapindaceae & 3 & 4 \\
\hline Sapotaceae & 1 & 1 \\
\hline Solanaceae & 1 & 1 \\
\hline Tiliaceae & 1 & 1 \\
\hline Ulmaceae & 1 & 1 \\
\hline Verbenaceae & 1 & 1 \\
\hline Violaceae & 1 & 1 \\
\hline TOTAL & 47 & 51 \\
\hline
\end{tabular}

O número crescente de espécies com dispersão zoocórica relacionado ao aumento de precipitação em diferentes florestas parece ser uma tendência em regiões tropicais e subtropicais como consta nos trabalhos de Gentry, Frankie et al. e Matthes apud MORELLATO (1991). Comparandose os resultados encontrados no Morro de Santo Antão com os de outros 
trabalhos: SILVA \& LONGHI (1989), MACHADO \& LONGHI (1990), BEARZI et al. (1992), VACCARO et al. (1992) e LONGHI (1993), realizados na Floresta Estacional Decidual percebe-se que a composição florística dos morros ao redor da cidade de Santa Maria é semelhante.

TABELA 3: Nome científico, familia e dispersão das espécies arbóreas encontradas na floresta do Morro de Santo Antão:

\begin{tabular}{lcc}
\hline \multicolumn{1}{c}{ NOME CIENTÍFICO } & FAMÍLIA & DISPERSÃO \\
\hline Acnistus breviflorus & Solanaceae & Zoocórica \\
Actinostemon concolor & Euphorbiaceae & Autocórica \\
Allophylus edulis & Sapindaceae & Zoocórica \\
Allophylus guaraniticus & Sapindaceae & Zoocórica \\
Bauhinia forficata & Caesalpiniaceae & Anemocórica \\
Carica quercifolia & Caricaceae & Zoocórica \\
Campomanesia xanthocarpa & Myrtaceae & Zoocórica \\
Casearia sylvestris & Flacourtiaceae & Zoocórica \\
Celtis iguanaea & Ulmaceae & Zoocórica \\
Chomelia obtusa & Rubiaceae & Zoocórica \\
Cordia trichotoma & Boraginaceae & Anemocórica \\
Chrysophyllum gonocarpum & Sapotaceae & Zoocórica \\
Cupania vernalis & Sapindaceae & Zoocórica \\
Dalbergia frutescens & Fabaceae & Anemocórica \\
Diospyros inconstans & Ebenaceae & Zoocórica \\
Enterolobium contortisiliquum & Mimosaceae & Autocórica \\
Erythrina falcata & Fabaceae & Autocórica \\
Eugenia involucrata & Myrtaceae & Zoocórica \\
Eugenia uniflora & Myrtaceae & Zoocórica \\
Ficus luschnathiana & Moraceae & Zoocórica \\
Helietta apiculata & Rutaceae & Anemocórica \\
Hybanthus biggibosus & Violaceae & Zoocórica \\
Lithraea molleoides & Anacardiaceae & Zoocórica \\
Luehea divaricata & Tiliaceae & Anemocórica \\
Machaerium stipitatum & Fabaceae & Anemocórica \\
Maclura tinctoria & Moraceae & Zoocórica \\
Matayba elaeagnoides & Sapindaceae & Zoocórica \\
Maytenus aquifolium & Celastraceae & Zoocórica \\
Myrcianthes pungens & Myrtaceae & Zoocórica \\
Myrocarpus frondosus & Fabaceae & Anemocórica \\
Nectandra megapotamica & Lauraceae & Zoocórica \\
Ocotea puberula & Lauraceae & Zoocórica \\
Parapiptadenia rigida & Mimosaceae & Anemocórica \\
\hline & & Continua ... \\
& & \\
& &
\end{tabular}

Rev. Ciência e Natura, Santa Maria, 22: 145 - 160 , 2000. 
TABELA 3: Conclusão...

\begin{tabular}{lcc}
\hline Patagonula americana & Boraginaceae & Anemocórica \\
Phytolacca dioica & Phytolaccaceae & Zoocórica \\
Plinia rivularis & Myrtaceae & Zoocórica \\
Prunus sellowii & Rosaceae & Zoocórica \\
Quillaja brasiliensis & Rosaceae & Anemocórica \\
Rollinia rugulosa & Annonaceae & Zoocórica \\
Schinus molle & Anacardiaceae & Zoocórica \\
Sebastiania commersoniana & Euphorbiaceae & Autocórica \\
Sorocea bonplandii & Moraceae & Zoocórica \\
Syagrus romanzoffiana & Arecaceae & Zoocórica \\
Tabebuia heptaphylla & Bignoniaceae & Anemocórica \\
Tecoma stans & Bignoniaceae & Anemocórica \\
Trichilia catigua & Meliaceae & Zoocórica \\
Trichilia claussenii & Meliaceae & Autocórica \\
Trichila elegans & Meliaceae & Autocórica \\
Vitex megapotamica & Verbenaceae & Zoocórica \\
Xylosma pseudosalzmannii & Flacourtiaceae & Zoocórica \\
Zanthoxylum rhoifolium & Rutaceae & Zoocórica \\
\hline
\end{tabular}

Pode-se observar que as familias mais importantes de todos os locais estudados são, praticamente, as mesmas. Leguminosae * e Myrtaceae são sempre as mais presentes em todos os locais da Floresta Estacional Decidual da Serra Geral. São também importantes deste tipo florestal, as famílias Lauraceae, Meliaceae, Sapindaceae, Euphorbiaceae e Moraceae. Estas cinco famílias sempre compõe mais de $50 \%$ do número total de espécies encontradas na floresta.

Em quase todos os locais já estudados da Floresta Estacional Decidual da Serra Geral predominam os gêneros Trichilia, Eugenia, Nectandra, Allophylus, Rapanea e Zanthoxylum. Embora todas as regiões da Floresta Estacional Decidual apresentam grande semelhança florística, principalmente, com relação às famílias botânicas ocorrentes, constata-se

* Para fins comparativos com trabalhos mais antigos Caesalpiniaceae, Fabaceae e Mimosaceae foram consideradas Leguminosae. 
sempre, que a composição de espécies ganha aspectos particulares a cada local estudado. Isso deve-se as características peculiares das diferentes regiões, como proximidade dos centros urbanos, grau de exploração madeireira, aspectos físicos e químicos do solo, entre outros.

No Morro de Santo Antão, analisado no presente trabalho, não foram encontradas as espécies Cedrela fissilis (cedro), Apuleia leiocarpa (grápia) e Cabralea canjerana (cangerana), e muito raramente alguns indivíduos de Ipê-roxo e Guajuvira, o que pode ser uma confirmação da ação predatória do homem, retirando estas madeiras de valor econômico madeireiro.

\section{CONSIDERAÇÕES FINAIS}

As famílias Leguminosae, Myrtaceae, Sapindaceae e Moraceae foram as mais importantes da comunidade.

Da mesma forma, os gêneros Trichilia, Eugenia e Allophylus foram os mais bem representados em número de espécies.

Os resultados do presente trabalho, revelaram que as espécies Sorocea bonplandii (cincho), Cupania vernalis (camboatá-vermelho), Matayba elaeagnoides (camboatá-branco), Actinostemon concolor (laranjeira-do mato), Schinus molle (aroeira-piriquita), Nectandra megapotamica (canela-preta), Trichilia claussenii (catiguá-vermelho), Allophylus edulis (chal-chal), entre outras, foram as mais freqüentes e características da floresta do Morro de Santo Antão.

A maioria das espécies apresentou síndrome de dispersão zoocórica, o que confirma a importância ecológica deste tipo de dispersão de sementes, para a manutenção da maioria das espécies arbóreas, bem como do equilíbrio entre a flora e a fauna da região. 


\section{REFERÊNCIAS BIBLIOGRÁFICAS}

BEARZI, R. C.; POLTRONIERI, V. C; LONGHI, S. J. Estrutura fitossociológica do Morro das Três Marias em Santa Maria, RS. In: CONGRESSO FLORESTAL ESTADUAL, 7., 1992, Nova Prata. Anais... Santa Maria: UFSM, 1992. p.376-394.

DIAS, C.A.; MELLO, S. C.; CASTAMAN, D.; ALVAREZ FILHO, A.; LONGHI, S.J.; DOROW, T.S. do C.; DELLA ZZANA, J.G.; VACCARO, S. Estudo florístico e fitossociológico do município de Santa Maria, RS. I etapa: Depressão Central - Morros Testemunha. In: SIMPÓSIO SOBRE ECOSSISTEMAS NATURAIS DO MERCOSUL - O AMBIENTE DA FLORESTA, 1., 1996, Santa Maria. Anais... Santa Maria: UFSM, CEPEF, 1996. p. 97-118.

FISCHER, K. E.; CHAPMAN, C. A. Frugivores and fruit syndromes; differences in patterns at the genus and species level. Oikos, v.66, n.3, p.472-482, 1993.

HOWE, H. F.; WESTLEY, L. C. Ecological relationships of plants and animals. Oxford: Univ. Press. Oxford, 1988. 768 p.

HUECK, K. As florestas da América do Sul. São Paulo: Polígono, 1972. $466 \mathrm{p}$.

KLEIN, R.M. Síntese ecológica da Floresta Estacional da Bacia do Rio Jacuí e importância do reflorestamento com essências nativas (RS). In: CONGRESSO FLORESTAL ESTADUAL, 5., 1984, Nova Prata. Anais... Porto Alegre: Secretaria de Estado da Agricultura - Prefeitura Municipal de Nova Prata, 1984. p.265-278.

LEMOS, R.C.; AZOLIN, M.D.; ABRAO, P.R.; SANTOS, M.C.L. Levantamento de reconhecimento dos solos do Estado do Rio Grande do Sul. Recife: Ministério da Agricultura- Departamento Nacional de Pesquisas Agro-pecuárias Divisão de Pesquisa Pedológica, 1973. 431p. (Boletim Técnico, 30).

LONGHI, S. J. Aspectos fitossociológicos comparativos da vegetação arbórea de quatro morros de Santa Maria, RS. Santa Maria: 1993. 179 p. Tese (Professor Titular) - Universidade Federal de Santa Maria. 
MACHADO, P, F. dos S.; LONGHI, S. J. Aspectos florísticos e fitossociológicos do "Morro do Elefante", Santa Maria, RS. Rev. Centro de Ciências Rurais, v.20, n.3-4, p.261-280, 1990.

MONTOYA MAQUIN, J .M.; MATOS, G. F. El sistema de Küchler: un enfoque fisionómico-estructural para la descripción de la vegetación. Turrialba, v.17, n.2, p.169-180, 1967.

MORELLATO, L.P.C. Estudo da fenologia de árvores, arbustos e lianas de uma floresta semi-decídua no sudeste do Brasil. Campinas: Instituto de Biologia-UEC. 1991. 176p. (Tese de Doutorado).

NASCIMENTO, A. R. T.; LONGHI, S. J. Diversidade florística e freqüência de síndromes de dispersão em um fragmento florestal, no município de Candelária-RS. In: FOREST'96, 1996, Belo Horizonte. Anais ..., Belo Horizonte: BIOSFERA, 1996. p. 200-202.

PIRES-O'BRIEN, M. J.; O'BRIEN, C.M. Aspectos evolutivos da fenologia reprodutiva das árvores tropicais. Belém: FCAP. Serviço de Documentação e Informação, 1995. 25 p.

RAMBO, R. A fisionomia do Rio Grande do Sul. Porto Alegre: Livraria Selbach, 1956. $456 \mathrm{p}$.

RIZZINI, C. T. Nota prévia sobre divisão fitogeográfica do Brasil. Rev. Bras. Geogr.,v.25, n.1, p.3-64, 1963.

SILVA, L. C. da; LONGHI, S. J. Aspectos florísticos e fitossociológicos do Morro do Link, em Santa Maria, Rio Grande do Sul. In: SEMANA DO AMBIENTE, 3., 1989, Piracicaba. Anais... Piracicaba: Centro de Energia Nuclear na Agricultura-Universidade de São Paulo, 1989. p.177-207.

STEBBINS, G.L. Flowering plants: Evolution above the species level. Cambridge: The Becknap Press of Harvard University Press. 1977. $397 p$.

TABARELLI, M.; MACHADO, P. F. dos S.; LONGHI, S. J. Aspectos florísticos de um trecho da mata ciliar do rio Ibicuí, nos municípios de Alegrete e São Francisco de Assis, RS. In: CONGRESSO FLORESTAL ESTADUAL, 7., 1992, Nova Prata. Anais... Santa Maria: UFSM, 1992. p.416-428. 
VACCARO, S.; HESS, A. F.; LONGHI, S. J. Estudo da composição florística e estrutura da vegetação arbórea da sede do município de Santa Tereza RS. In: CONGRESSO FLORESTAL ESTADUAL, 7, 1992, Nova Prata. Anais... Santa Maria: UFSM, 1992. p.395-415.

VAN DER PIJL, L. Principles of dispersal in higher plants. 3.ed. Berlin: Springer-Verlag, 1982. $213 \mathrm{p}$.

VELOSO, H. P.; RANGEL FILHO, A. L. R.; LIMA, J. C. A. Classificação da vegetação brasileira, adaptada a um sistema universal. Rio de Janeiro: IBGE/PROJETO RADAMBRASIL, 1991. 123 p. 